\title{
Communicable Diseases Report, NSW, May and June 2012
}

\author{
Communicable Diseases Branch \\ NSW Department of Health
}

For updated information, including data and facts on specific diseases, visit www.health.nsw.gov.au and click on Public Health and then Infectious Diseases. The communicable diseases site is available at: http://www.health.nsw.gov.au/ publichealth/infectious/index.asp.

Figure 1 and Tables 1 and 2 show notifications of communicable diseases received in May and June 2012 in New South Wales (NSW).

\section{Enteric infections \\ Outbreaks of suspected foodborne disease}

In March, NSW Health were notified of a complaint regarding a banquet at a Sydney restaurant (restaurant A). Eighteen people from a group of 24 who dined at the restaurant developed gastrointestinal symptoms following the meal. An epidemiological investigation was conducted after four of these people who became ill returned stool samples which tested positive for Salmonella (Salmonella Typhimurium MLVA 3-9-9-12-523: STm strain A). All those people who became ill had consumed the same dessert, a Bombe Alaska, containing raw egg meringue. NSW Food Authority officers inspected restaurant A and requested that it cease serving this dessert as it contained raw egg. The eggs used at restaurant $\mathrm{A}$ were purchased from an egg farm (egg farm A). This farm was inspected in April 2012. This egg farm also supplied another 10 businesses and sold eggs directly to the public.

Salmonella (STm strain A) was previously uncommon in NSW, but from January to March 2012 it was one of the most commonly reported types among cases with salmonellosis in south west Sydney. STm strain A was also identified in three case-patients who had complained of illness after a party at an unrelated restaurant in Sydney in January 2012. At the time, that investigation did not identify an association between illness and any particular food item. However, a profiterole cake consumed at the party had been supplied by a bakery in the area (bakery A). Bakery A was listed as one of the wholesale customers of egg farm A. The NSW Food Authority inspected bakery A in May 2012 and the same STm strain A was identified on re-usable piping bags, machine nozzles and from a bowl of freshly whipped cream.

NSW Health and OzFoodNet epidemiologists then interviewed additional confirmed cases of STm strain A (27 case-patients) and of another closely related strain (MLVA 3-9-8-12-523: STm strain B) (30 case-patients) that had been notified between January and April to determine whether they had consumed food prepared by either restaurant $\mathrm{A}$ or bakery $\mathrm{A}$, from any of the other businesses that egg farm A supplied, or whether they had bought eggs directly from egg farm A. Despite the delay from the time of their illness to being interviewed, $46 \%$ (30/65) case-patients reported consuming eggs from either restaurant A or bakery $\mathrm{A}$ in their incubation period and a further case reported purchasing eggs directly from the farm. The NSW Food Authority are working with farm A to reduce the possibility of contamination of eggs occurring in the future.

\section{Respiratory infections \\ Influenza}

Influenza activity increased markedly in May and June 2012, consistent with an early start to the winter influenza season. Increased activity was measured by: increased notifications of laboratory-confirmed cases; an increased proportion of respiratory tests positive for influenza; outbreaks of influenza in aged-care facilities; and increased numbers of people presenting to 59 of the state's largest emergency departments with influenza-like illness and pneumonia, especially people aged over 65 years.

Overall, activity was well above that seen in the past 2 years for this time of year but remained below the peak levels seen during the influenza pandemic in 2009 and the severe influenza season in 2007.

Laboratory testing data for influenza showed that the most common influenza strain circulating was the new A/Victoria/361/2011-like strain, although influenza B viruses continued to circulate at low levels. No influenza antiviral resistance was reported.

The WHO Collaborating Centre on Influenza has advised that current influenza vaccines are likely to induce 
significant protection against the new H3N2 strain, and that the current B strain is well matched to the vaccine strain.

For a more detailed report on respiratory activity in NSW see: http://www.health.nsw.gov.au/PublicHealth/ Infectious/influenza_reports.asp

\section{Vaccine-preventable diseases \\ Meningococcal disease}

Twelve cases of meningococcal disease were notified in NSW in May and June 2012 (six in May and six in June), an increase from nine for the same period in 2011. The age of the case-patients ranged from 2 months to 65 years and included five children aged under 5 years. There were no deaths notified in this period. Seven cases were due to serogroup B (for which there is no vaccine), one was due to serogroup Y, two were unable to be typed, and for two there was insufficient specimen collected.

Of the nine cases notified during the same period in 2011 , five were due to serogroup B, one to serogroup $\mathrm{Y}$ and the remaining three were of an undetermined serogroup.

It is recommended that a single dose of the vaccine for meningococcal $\mathrm{C}$ disease be given to all children at the age of 12 months as well as to people at high risk of the disease. $^{1}$

\section{Measles}

Fifteen cases of measles were notified in NSW in May and June 2012. This was an increase from six cases reported for the same period in 2011 .

One case of measles notified during this period was epidemiologically linked to a previously reported cluster that commenced in April and was associated with a young adult returning home from Thailand while infectious (total case-patients, $n=4$ ).

A cluster of six cases notified during May and June were linked to the emergency department of the Children's Hospital at Westmead and subsequently at a high school formal. The average age of case-patients was 11 years (range: 7 months to 32 years). Of the six case-patients, three were too young to be vaccinated, two were unsure of their vaccination status and one reported receiving only one measles-containing vaccine. Cases in this cluster were notified across metropolitan Sydney.

A second cluster of six cases notified during May and June were reported primarily in under-immunised teenage students at a high school in the Sydney South West Local Health District. The case-patients were aged between 13 and 19 years. Of the six case-patients, three were not vaccinated, two were unsure of their vaccination status and one reported receiving only one measles-containing vaccine.

A further two sporadic measles cases were also notified during this period. The first case was an unvaccinated 1year old infant who acquired their infection in China. The second case was a 7-month old infant (who was too young to be vaccinated) whose source of infection remains unknown (measles virus genotype B3). While the measles virus genotype B3 has been the predominant genotype identified in African countries in recent years, and to a lesser degree in other continents, ${ }^{2}$ it has not been identified during the past decade in NSW. Given the high sensitivity of measles surveillance in NSW and the lack of previously identified measles virus genotype B3, this suggests the infant's infection was transmitted from an unknown person who acquired their infection overseas. No further transmission was identified from either of these sporadic cases.

While a public health investigation did not identify a direct epidemiological link between the Thailand cluster or the two clusters reported during May and June, the clusters were found to have the same measles virus genotype and sequence of D8. In the absence of an epidemiological linkage between the clusters, the genotype information indicates that it is likely that there have been further undiagnosed (and therefore un-notified) cases of measles in the community.

Under-immunised high school students and infants too young to be vaccinated have been at the greatest risk of disease in these measles clusters. Two doses of measlesmumps-rubella (MMR) vaccine at 12 months and at 4 years of age are recommended for all children.

\section{Pertussis (whooping cough)}

During May and June 2012, 962 cases of pertussis were notified in NSW. This is a decrease from the 2036 cases notified for the same period in 2011 .

NSW Health recently announced a refocused strategy to help prevent severe disease and death from pertussis by offering free vaccination to new mothers in maternity units or at their GP within 2 weeks of giving birth. ${ }^{3}$ The program will continue until more definitive evidence becomes available about the effectiveness of vaccinating adults to protect new babies. NSW Health is collaborating with the National Centre for Immunisation Research and Surveillance in a study to assess the effectiveness of this strategy.

Direct protection for young infants remains available through free vaccination that is administered at 2, 4 and 6 months of age. The first dose can be provided as early as 6 weeks of age. There is also a booster dose at $3 \frac{1}{2}$ to 4 years of age. 


\section{Sexually transmissible infections/bloodborne viruses \\ HIV infections}

In 2011, 330 people were newly diagnosed with HIV infection in NSW, an increased number than in 2010 $(n=307)$, but similar to the previous 3-year average of 320. Of those newly diagnosed in 2011, 309 (94\%) were male and the median age at diagnosis was 35 years. The probable risk exposure category of most of the casepatients was reported to be homosexual contact $(n=277$, $84 \%$ ), with the exposure category of the others reported to be: heterosexual contact ( $n=42,13 \%)$, injecting drug use $(n=8,2.4 \%)$ and other/unknown source $(n=3,0.9 \%)$.

The median age of homosexually-acquired HIV casepatients was 34 years and more than half of these people $(n=163,59 \%)$ were Australian-born. Three-quarters (73\%) of homosexually-acquired infections were thought to be acquired in Australia, while 14\% were reported to have been acquired overseas. There was an increase in both the number and proportion of homosexually-acquired HIV infections notified in $2011(n=277,84 \%)$ compared to $2010(n=235,77 \%)$.

Of the 42 heterosexually-acquired cases, 26 (67\%) were male and $16(43 \%)$ were female. The median age was 40 years. Fifteen case-patients (36\%) were born in Australia, $12(29 \%)$ were born in high HIV prevalence countries, ${ }^{4}$ $14(33 \%)$ were born in countries not classified as having a high prevalence of HIV infection and there was one $(2.4 \%)$ case-patient for whom the country of birth was not stated.
Of the eight case-patients whose likely risk exposure was injecting drug use, four were men and four were women. The median age of this group was 41 years.

Promoting safe sex practices, particularly among men with homosexual exposures, remains important in the prevention of HIV infection.

A summary of 2011 HIV surveillance data has been published on the NSW Health website at http:// www.health.nsw.gov.au/resources/publichealth/infectious/ diseases/hiv_aids/new_diagnoses_hiv_infection_2011.pdf and http://www.health.nsw.gov.au/resources/publichealth/ infectious/diseases/hiv_aids/hiv_surveillance_data_2011. pdf.

\section{References}

1. National Health and Medical Research Council. The Australian Immunisation Handbook. 9th ed. Canberra: Australian Government Department of Health and Ageing; 2008.

2. Rota PA, Brown K, Mankertz A, Santibanez S, Shulga S, Muller CP et al. Global distribution of measles genotypes and measles molecular epidemiology. J Infect Dis 2011; 204(Supplement 1): S514-23. doi:10.1093/infdis/jir118

3. NSW Health. New Strategy To Control Whooping Cough Spread In NSW. [Internet]. Available at: http://www.health.nsw. gov.au/news/2012/20120622_00.html (Cited 9 July 2012).

4. Joint United Nations Programme on HIV/AIDS (UNAIDS). Report on the global AIDS epidemic 2010. UNAIDS; 2010. Available at: http://www.unaids.org 
Figure 1. Reports of selected communicable diseases, NSW, Jan 2004 to June 2012, by month of onset

Preliminary data: case counts in recent months may increase because of reporting delays.

Laboratory-confirmed cases only, except for measles, meningococcal disease and pertussis.

$\mathrm{BFV}=$ Barmah Forest virus infections, RRV $=$ Ross River virus infections,

lab conf = laboratory confirmed,

Men $\mathrm{Gp} C$ and $\mathrm{Gp} B=$ meningococcal disease due to serogroup $C$ and serogroup $B$ infection, other/unk = other or unknown serogroups.

NB: Multiple series in graphs are stacked, except gastroenteritis outbreaks.

NB: Outbreaks are more likely to be reported by nursing homes and hospitals than by other institutions.

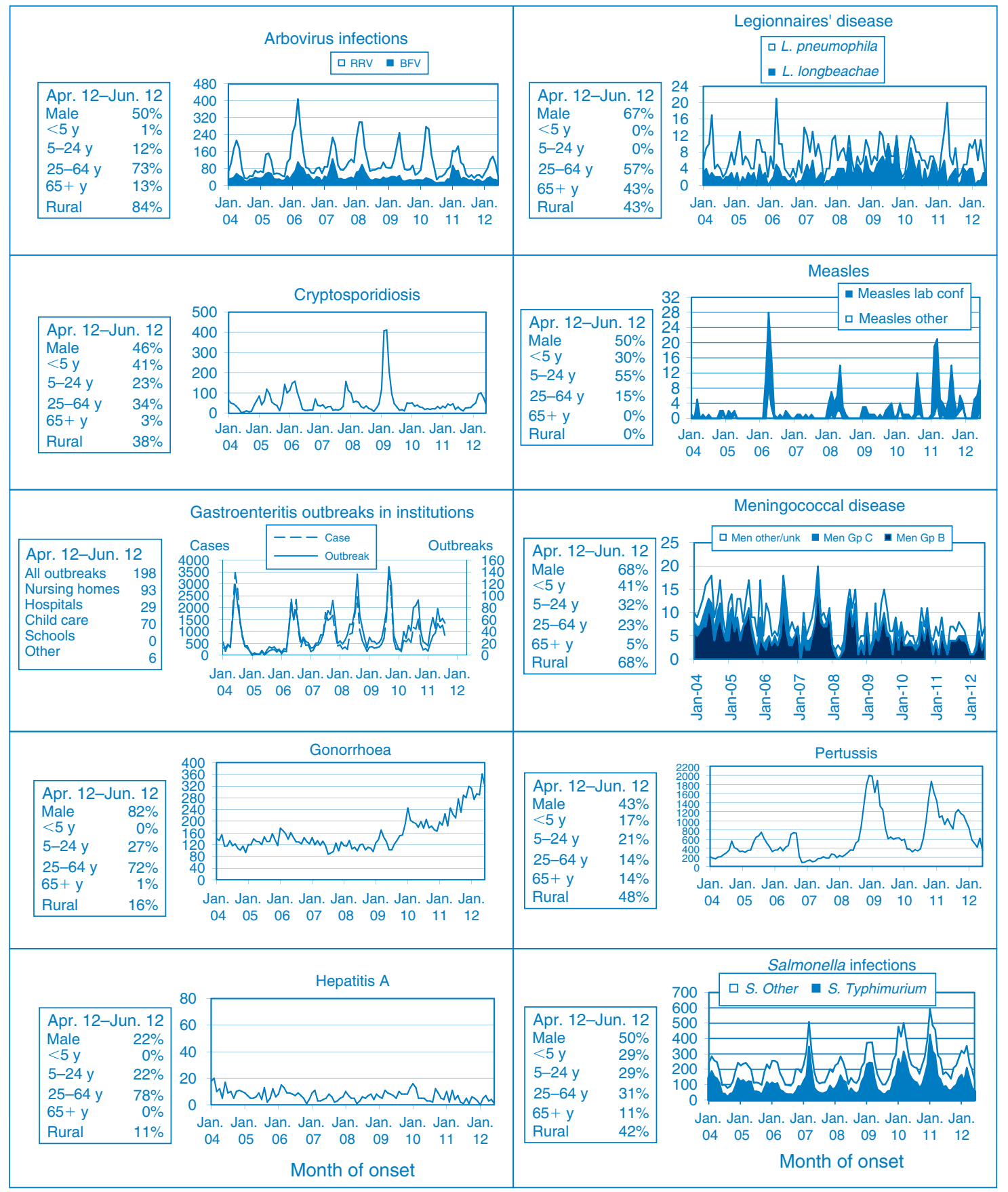




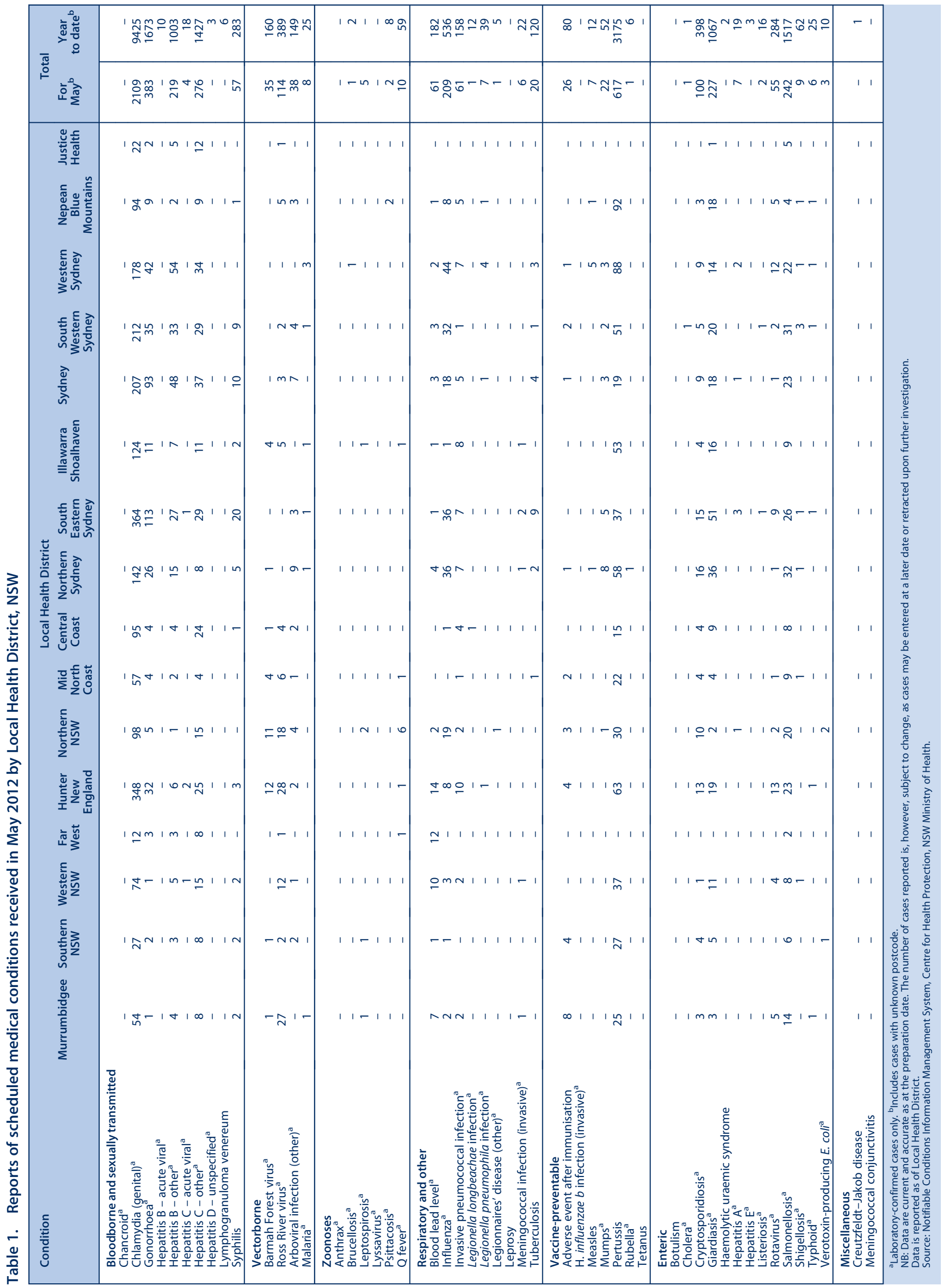




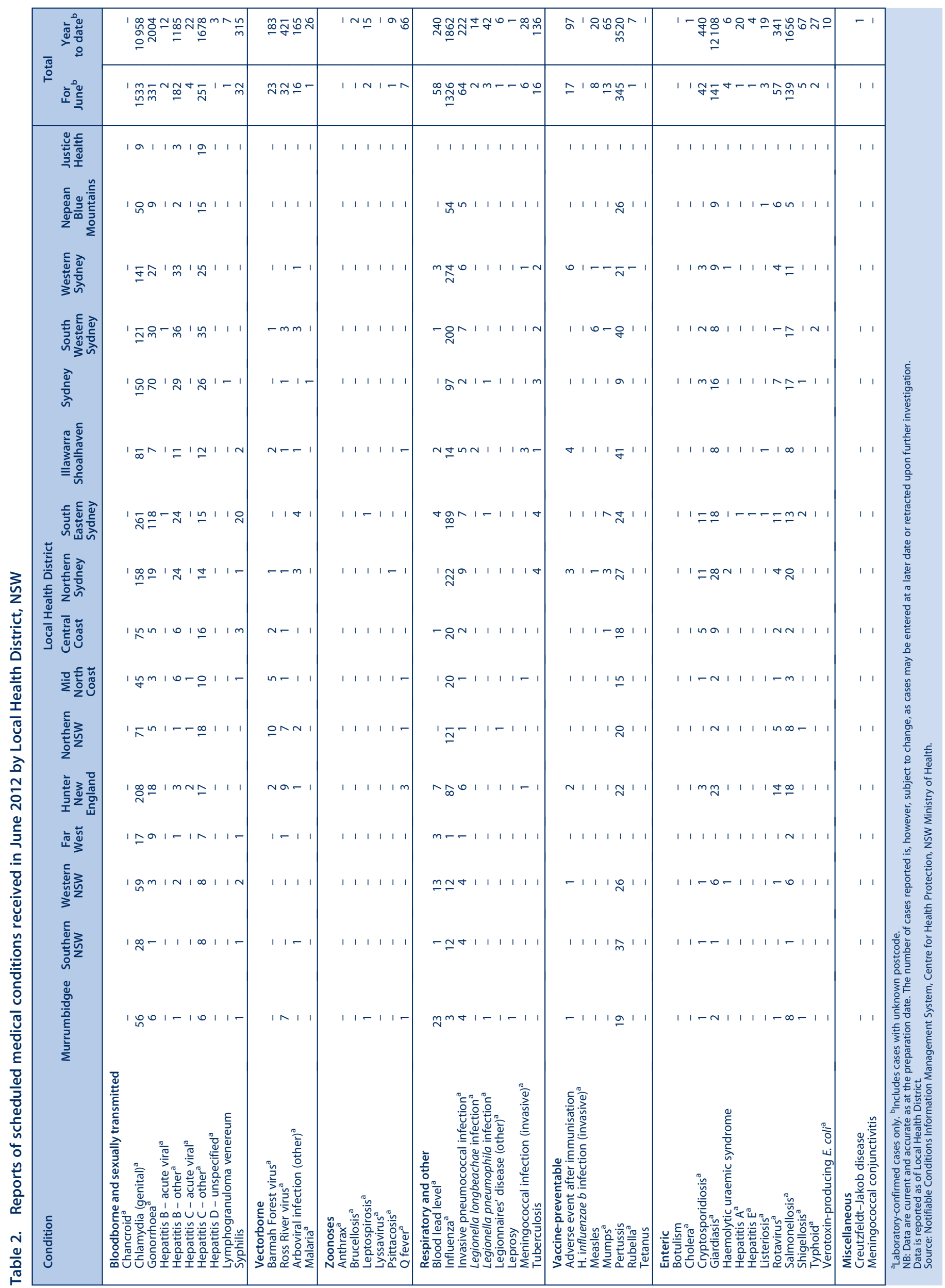

